\title{
Effects of Mind-Body Movements on Balance Function in Stroke Survivors: A Meta-Analysis of Randomized Controlled Trials
}

\author{
Liye Zou ${ }^{1,2, *(\mathbb{D})}$, Albert Yeung ${ }^{3}$, Chunxiao Li ${ }^{4}$, Shin-Yi Chiou ${ }^{5}$, Nan Zeng ${ }^{6}$ (D), \\ Huey-Ming Tzeng ${ }^{7} \mathbb{B}^{D}$, Lin Wang ${ }^{1, *}$, Zhanbing Ren ${ }^{8}$, Taquesha Dean ${ }^{3}$ and \\ Garrett Anthony Thomas ${ }^{3}$ \\ 1 Department of Physical Education, Wuhan University of Technology, Wuhan 430070, China \\ 2 Department of Sports Science and Physical Education, the Chinese University of Hong Kong, Shatin, \\ Hong Kong, China \\ 3 Depression Clinical and Research Program, Harvard Medical School, Harvard University, Boston, MA 02114, \\ USA; AYEUNG@mgh.harvard.edu (A.Y.); TDEAN4@mgh.harvard.edu (T.D.); \\ GTHOMAS12@mgh.harvard.edu (G.A.T.) \\ 4 Department of Health and Physical Education, The Education University of Hong Kong, Tai Po, Hong Kong, \\ China; cxli@eduhk.hk \\ 5 School of Sport, Exercise and Rehabilitation Sciences, University of Birmingham, Birmingham B15 2TT, UK; \\ s.chiou12@imperial.ac.uk \\ 6 School of Kinesiology, University of Minnesota-Twin Cities, Minneapolis, MN 55455, USA; \\ zengx185@umn.edu \\ 7 College of Nursing, University of Saskatchewan, 104 Clinic Place, Saskatoon, SK S7N 2Z4, Canada; \\ tzenghm@gmail.com \\ 8 Department of Physical Education, Shenzhen University, Shenzhen 518060, China; rzb@szu.edu.cn \\ * Correspondence: wanglin123@126.com (L.W.); liyezou123@cuhk.edu.hk (L.Z.)
}

Received: 26 May 2018; Accepted: 17 June 2018; Published: 20 June 2018

\begin{abstract}
Objective: We performed a systematic review with meta-analysis and meta-regression to determine if mind-body movements (MBM) could be effective in rehabilitating balance function among stroke survivors. Methods: A literature search was conducted using major Chinese and English electronic databases from an inception until January 2018. Randomized controlled studies were included in our meta-analysis. Data was independently extracted by two review authors using a pre-developed table and confirmed by a third party to reach a consensus. Pooled effect size (Hedge's g) was computed while the random-effect model was set. Results: The meta-analytic results showed a significant benefit of the MBM intervention on increased balance function compared to the control groups (Hedge's $g=1.59, \mathrm{CI} 0.98$ to $2.19, p<0.001, I^{2}=94.95 \%$ ). Additionally, the meta-regression indicated that the total number of sessions ( $\beta=0.00142,95 \%$ CI 0.0039 to $0.0244, p=0.0067)$ and dose of weekly training ( $\beta=0.00776,95 \%$ CI 0.00579 to $0.00972, p=0.00$ ) had significantly positive effects on balance function. Conclusions: The study encouraging findings indicate the rehabilitative effect of a MBM intervention for balance function in stroke survivors. However, there were significant limitations in the design among several of the included trials. Additional studies with more robust methodologies are needed to provide a more definitive conclusion.
\end{abstract}

Keywords: Tai Chi; Yoga; mindfulness movement; stroke; rehabilitation

\section{Introduction}

Strokes are the second-leading cause of death worldwide and are considered one of the most common causes of long-term disabilities [1]. According to the World Health Organization (WHO), 
approximately 15 million individuals worldwide experience a first-ever stroke each year [2]. Of these, there are an estimated five million stroke survivors who are left permanently disabled [2], placing a burden on family and society. The social-economic costs (such as medications, hospitalization, job loss/reduced productivity) of Chinese stroke survivors were $\$ 16.08$ billion in 2008 and this number continues to increase [3]. Furthermore, approximately $80 \%$ of the stroke survivors suffer from balance disorders due to visually guided performance deficit, loss of proprioception, and altered walking patterns $[4,5]$. Compared to healthy age-matched adults, stroke survivors with impaired balance are seven times more likely to fall, which is associated with a higher risk of fractures, ill-being, anxiety, depression, and even mortality $[6,7]$.

According to the Centers for Disease Control and Prevention, $67 \%$ of the stroke survivors require treatments to improve their balance [8,9]. To help them attain the best possible quality of life, physical therapy is commonly prescribed as the main approach to pharmacotherapy to help stroke survivors regain motor function and the ability to control their balance [10,11]. However, using physical therapy to improve balance requires time-consuming input from therapists while they carry out the interventions. In addition, it is usually limited to authorized rehabilitation centers which are not always affordable or accessible by stroke survivors [12]. Therefore, it is crucial to identify complementary approaches for balance rehabilitation for stroke survivors that are low cost and community-based, with the goal of maximizing the recovery of balance function post-stroke.

Mind-body movements (e.g., Tai Chi, yoga or Qigong) may be a complementary rehabilitation method for balance rehabilitation in stroke survivors [13-15], since they share similar mechanisms of movement that align with conventional rehabilitation methods, such as the Bobath technique (focusing on quality of movement and correct movement patterns to strengthen kinesthetic awareness, leading to better postural stability) and proprioceptive neuromuscular facilitation [16]. Smooth and well-coordinated whole body movements in these mind-body movements (MBM) routines assist with the control of voluntary weight-shifting, which could potentially strengthen the core and lower limb muscles of stroke survivors, therefore naturally leading to better balance performance $[17,18]$. Practicing MBM also requires musculoskeletal relaxation when integrated with breath control and mental focus, which could lead to decreased fatigue and increased patient adherence to achieve faster recovery $[19,20]$.

Recently, a number of empirical studies have been conducted that investigate the rehabilitative effects of MBM on balance within stroke survivors [21-27]. Subsequently, review studies have emerged on this topic; however, based on qualitative analyses, all of the reviews have only paid attention to Tai Chi or yoga [28-31]. Furthermore, most of the reviews do not include Chinese academic databases due to the language barrier. Moreover, there have been increasing data from the emerging literature showing that Tai Chi and Qigong have rehabilitative effects on balance function in stroke survivors. Therefore, a meta-analytic systematic review is needed to critically assess available evidence concerning the effects of these three MBM on balance function in stroke survivors. We expect that the updated findings of this review could allow scholars and health professionals to design and develop effective MBM rehabilitative protocols that can speed up the balance recovery process and maximize treatment effects.

\section{Methods}

\subsection{Protocol and Registration}

This review was carried out in accordance with the Preferred Reporting Items for Systematic Review and Meta-Analysis (PRISMA) guidelines and the checklist was completed [32]. The project was registered with the International Prospective Register of Systematic Reviews (No. CRD4201808 5213) to eliminate duplicates. 


\subsection{Information Sources and Search}

The leading reviewer (L.Z.) performed the literature search (January 2018) using both English (e.g., PubMed, Physiotherapy Evidence Database, Cochrane Library, and WHO International Clinical Trials Registry Platform) and Chinese (e.g., China National Knowledge Infrastructure, Wanfang, and Chinese Clinical Trial Registry) electronic databases without restricting publication dates. Two groups of search terms were used in a combined manner: (1) "stroke", "cerebrovascular accident", "brain attack", "brain ischemia", or "intracranial hemorrhage"; and (2) "Yoga", "Tai Chi/Taiji”, or "Qigong”. Relevant studies were also manually identified based on cross-referencing searches.

\subsection{Eligibility Criteria and Study Selection}

Studies that met the following criteria were included: (1) was a randomized controlled trial (RCT); (2) were published in peer-reviewed Chinese/English journals; (3) given that yoga, Tai Chi, and Qigong are ranked as the top 3 complementary therapies according the National Health Interview Survey [33], usage of at least one of these three MBM as the primary interventions for at least 2 weeks; (4) samples of at least 15 stroke survivors; and (5) reporting of quantitative data for computing the standardized training effect (Hedge's $g$ ) on balance function. Controlled trials without randomization, cross-sectional studies, case reports/series, and review studies were excluded. Two researchers (L.Z. and N.Z.) independently screened the title and abstract and the full text was retrieved when necessary. If there was disagreement between the two researchers, a third reviewer (A.Y.) was involved in the discussion until a consensus was reached.

\subsection{Data Items and Collection Processes}

Researchers (L.Z. and N.Z.) used a pre-developed table (Table 1) for extracting data items which included references (i.e., name of the leading author, year of publication, and study location), participant characteristics (initial sample size and attribution rate, mean age/age range, course of disease, and stroke type, i.e., ischemic or hemorrhage), intervention components (types, training frequency and length, mode of combination, training mode, weekly training dosage of at least 150 min weekly, which, per the National Physical Activity Guidelines, is recommended for persons with physical disabilities) [34], total number of training sessions, control group activity (treatment mode), and the balance/instrument used. Two researchers (L.Z. and N.Z.) independently extracted data from the eligible studies. The data entry was then cross-checked by a third party (A.Y.).

\subsection{Risk of Bias in Individual Studies}

Based on previous studies [35,36], we adapted the Physiotherapy Evidence Database (PEDro) scale to assess risk of bias in the eligible studies. Given that the blinding of participants and instructor(s) are impractical in non-pharmacological interventions, we removed the two items that assessed that. The updated PEDro scale consisted of nine items: randomization, concealed allocation, similar baseline, blinding of assessors, more than $85 \%$ retention, missing data management/intention-to-treat analysis, between-group comparison, point measure of variability, and co-intervention, which should be either avoided in the trial design or similar between the index and control groups. Points were only awarded if authors explicitly reported the information. Each study could have earned a maximum of nine points, with higher scores indicating lower risk of bias.

\subsection{Synthesis of Results, Risk of Bias across Studies, and Additional Analysis}

Effect sizes (Hedge's $g$ ) across individual studies were pooled using the random-effect model in a Comprehensive Meta-Analysis Software. The value of I-squared was used to determine the heterogeneity of the effect sizes across the RCTs selected: (1) small $=25 \%$; (2) medium $=50 \%$; (3) and large $=75 \%$. A series of sub-group analyses were conducted and analyzed, including intervention duration ( $<12$ weeks vs. $\geq 12$ weeks), training frequency ( $<5$ sessions/week vs. $\geq 5$ sessions/week), 
session length ( $<45 \mathrm{~min}$ vs. $\geq 45 \mathrm{~min}$, which according to General Guidelines for Exercise Training and Progression for Stroke Recovery and Rehabilitation, $45 \mathrm{~min}$ of exercise training is tolerable) [37], mode of combination (separate vs. simultaneous), weekly training duration of at least $150 \mathrm{~min}$ (yes vs. no), and total sample size (less than 60 vs. $60+$ ) were conducted. A meta-regression analysis was conducted with the total number of training sessions and dose of weekly training as the predictors. Finally, we used the funnel plot and Egger's regression intercept test to determine whether publication bias existed.

\section{Results}

\subsection{Study Selection}

Figure 1 shows the procedures of study selection. The electronic and manual searches resulted in 307 articles. Based on the titles of initially identified articles, there were 269 duplicates and therefore they were removed. After removing the 269 duplicates, 38 studies remained for screening. After reading abstract of the remaining 38 articles, seven irrelevant records were excluded. Based on the eligibility criteria, 13 studies were removed because they were either not RCTs $(n=3)$, were review studies $(n=5)$, had no MBM intervention $(n=2)$, provided no outcome of interest $(n=1)$, or had no data reported for analysis $(n=1)$.This left only 18 eligible RCTs to be included for the meta-analysis.

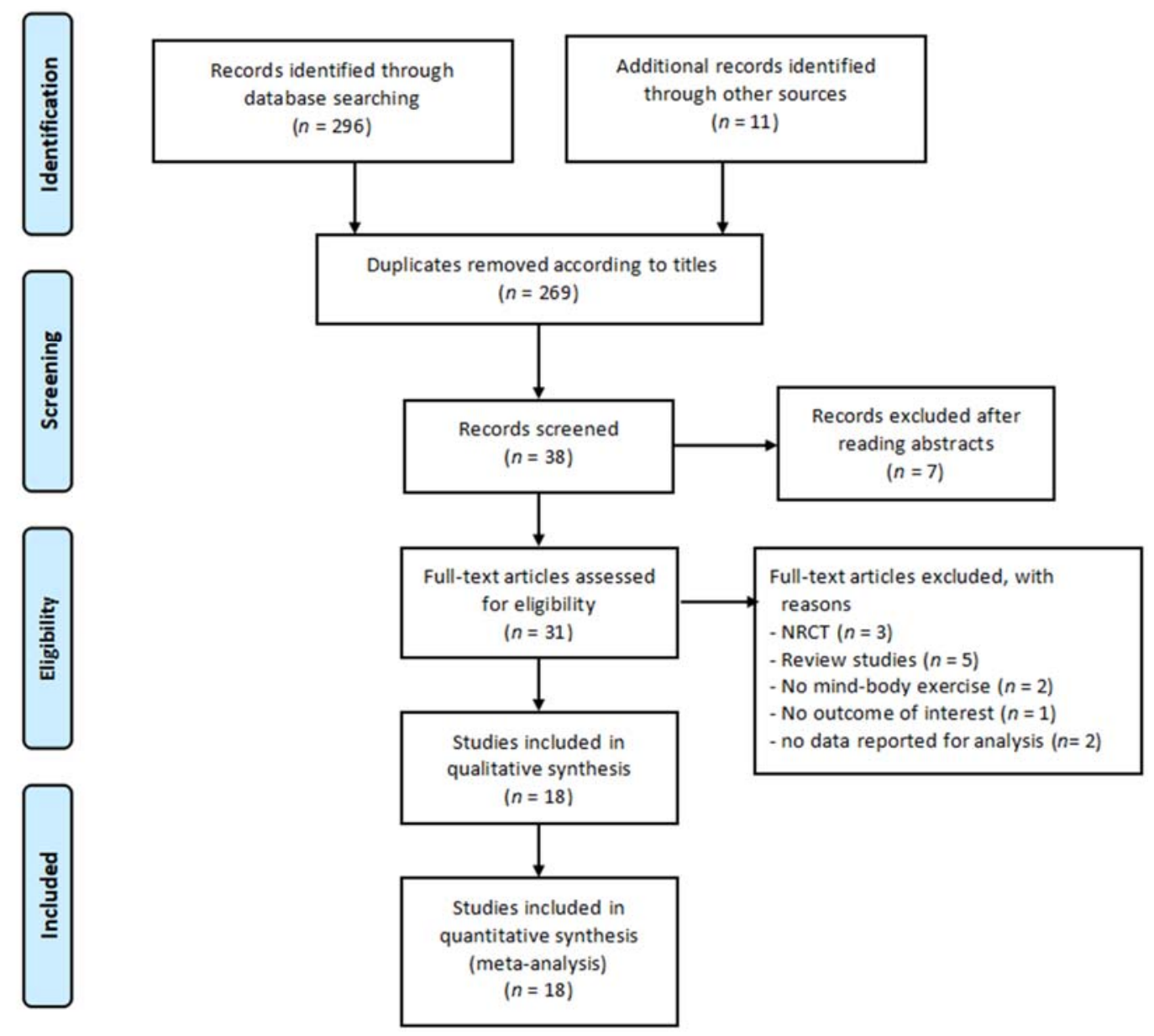

Figure 1. Procedures of study selection. (Note: NRCT means non-randomized control trial.) 


\subsection{Study Characteristics}

The characteristics of the 18 included studies are presented in Table 1. These studies were published between 2009 and 2017. Most of them were conducted in mainland China, followed by the USA $(n=2)$, Austria $(n=1)$, Japan $(n=1)$, and South Korea $(n=1)$. The total number of participants was $1189(\mathrm{MBM}=594$ and Control $=595)$, and the study's sample sizes varied from 22 to 224 , with attrition rates ranging from $1.3 \%$ to $21 \%$. The mean age of participants varied from 42.8 to 77.59 years old (range between 33 and 82 years). Both acute and chronic strokes were included and the average duration of disease was between 14.5 and 47.34 days, 6.22 and 7.01 weeks, or 1 to 81.6 months. The number of patients with ischemic $(n=415)$ and hemorrhage strokes $(n=231)$ were also reported in some RCTs. The intervention duration ranged from 4 to 12 weeks, with 1 to 7 sessions per week, each lasting 15 to $90 \mathrm{~min}$ per session. Only 11 studies reported weekly training sessions that lasted more than $150 \mathrm{~min}$. The total number of training sessions ranged between 8 and 84 sessions, with a total weekly training dose of 90 to $330 \mathrm{~min}$. Tai Chi was the most frequently used intervention $(n=12)$, followed by Qigong $(n=4)$, and yoga $(n=2)$. In $77.78 \%$ of the studies selected, the MBM intervention was combined with either balance training, educational programming, Bobath techniques and drug therapy, general rehabilitation and physical therapy, device-assisted rehabilitation, Prokin balance feedback training, or the usual treatment routine. The three different training modes were individual practice, group-based practice, and mixed mode. Adverse events, such as death, hospitalizations, falls, or injuries did not occur during MBM intervention. 
Table 1. Characteristics of studies selected.

\begin{tabular}{|c|c|c|c|c|c|c|c|c|c|c|c|}
\hline \multirow{2}{*}{$\begin{array}{l}\text { Reference } \\
\text { (Country) }\end{array}$} & \multicolumn{4}{|c|}{ Participant Characteristics } & \multicolumn{5}{|c|}{ Mind-Body Intervention } & \multirow{2}{*}{$\begin{array}{l}\text { Control Group } \\
\text { Activity }\end{array}$} & \multirow{2}{*}{$\begin{array}{c}\text { Outcomes } \\
\text { (Instrument) }\end{array}$} \\
\hline & $\operatorname{ISZ}(\mathrm{AT})$ & $\begin{array}{c}\text { Mean Age or Age } \\
\text { Range (yr) }\end{array}$ & Course of Disease & $\begin{array}{l}\text { Ischemicl } \\
\text { Hemorrhage }\end{array}$ & $\begin{array}{l}\text { Training Frequency and Length } \\
\text { (MB Component) }\end{array}$ & $\begin{array}{c}\text { Mode of } \\
\text { Combination }\end{array}$ & $\begin{array}{c}\text { Training } \\
\text { Mode }\end{array}$ & $\begin{array}{l}\text { (Training Dose) } \\
\geq 150 \text {-min weekly }\end{array}$ & $\begin{array}{l}\text { No. of Sessions } \\
\text { (Total Volume) }\end{array}$ & & \\
\hline $\begin{array}{l}\text { Taylor-Piliae [27], } \\
\text { USA }\end{array}$ & $\begin{array}{l}145(10 \%) \\
\text { MB: } 53 \\
\text { CG1: } 44 \\
\text { CG2: } 48\end{array}$ & $\begin{array}{l}\text { MB: } 71.5(10.3) \\
\text { CG1: } 69.6(9.4) \\
\text { CG2: } 68.2(10.3)\end{array}$ & $\begin{array}{c}\text { MB: } 39(40.2) \mathrm{M} \\
\text { CG1: } 33(58.7) \mathrm{M} \\
\text { CG2: } 38.7(46.7) \mathrm{M}\end{array}$ & $\begin{array}{l}\text { MB: } 33 / 12 \\
\text { CG1: } 32 / 8 \\
\text { CG2: } 30 / 14 \\
\text { 16 unknown }\end{array}$ & $\begin{array}{l}60 \mathrm{~min} \times 3 \text { sessions } / \mathrm{wk}, \\
12 \text { wks (24-style Tai Chi) }\end{array}$ & Separate & Group & (180 $\mathrm{min})$ Yes & $36(2160 \mathrm{~min})$ & $\begin{array}{l}\text { CG1: strength and } \\
\text { range of } \\
\text { movement } \\
\text { exercises; } \\
\text { CG2: phone call } \\
\end{array}$ & Balance (TBT) \\
\hline $\begin{array}{l}\text { Taylor-Piliae et al. [28], } \\
\text { China }\end{array}$ & $\begin{array}{l}28(11 \%) \\
\text { MB: } 16 \\
\text { CG: } 12 \\
\end{array}$ & $\begin{array}{l}\text { MB: } 72.8(10.1) \\
\text { CG: } 64.5(10.9)\end{array}$ & $\begin{array}{l}\text { MB: } 58.3(46.7) \mathrm{M} \\
\text { CG: } 47.9(42.5) \mathrm{M}\end{array}$ & $\begin{array}{l}\text { MB: } 12 / 4 \\
\text { CG: } 9 / 3\end{array}$ & $\begin{array}{l}60 \mathrm{~min} \times 3 \text { sessions } / \mathrm{wk}, \\
12 \mathrm{wks} \text { (24-style Tai Chi) }\end{array}$ & Separate & Group & (180 $\mathrm{min})$ Yes & $36(2160 \mathrm{~min})$ & Usual treatment & Balance (TBT) \\
\hline $\begin{array}{l}\text { Schmid et al. [29], } \\
\text { USA }\end{array}$ & $\begin{array}{l}47(9 \%) \\
\text { MB: } 37 \\
\text { CG: } 10\end{array}$ & $\begin{array}{l}\text { MB: } 63.9(8.7) \\
\text { CG: } 60.2(8.9)\end{array}$ & $\begin{array}{l}\text { MB: } 54.9(43.2) \mathrm{M} \\
\text { CG: } 36.4(23.6) \mathrm{M}\end{array}$ & $\begin{array}{l}\text { MB: } 26 / \mathrm{NR} \\
\text { CG: } 5 / \mathrm{NR}\end{array}$ & $\begin{array}{c}60 \mathrm{~min} \times 2 \text { sessions } / \mathrm{wk} \\
8 \mathrm{wks} \text { (Yoga) }\end{array}$ & $\begin{array}{l}\text { Simultaneous } \\
\text { (Usual treatment) }\end{array}$ & Group & $(120 \mathrm{~min})$ No & $16(960 \mathrm{~min})$ & Usual treatment & Balance (BBS) \\
\hline $\begin{array}{l}\text { Immink et al. [30], } \\
\text { Austria }\end{array}$ & $\begin{array}{l}25(12 \%) \\
\text { MB: } 12 \\
\text { CG: } 13\end{array}$ & $\begin{array}{l}\text { MB: } 56.1(13.6) \\
\text { CG: } 63.2(17.4)\end{array}$ & $\begin{array}{l}\text { MB: } 81.6(77.5) \mathrm{M} \\
\text { CG: } 23.3(12.5) \mathrm{M}\end{array}$ & NR & $\begin{array}{c}90 \mathrm{~min} \times 1 \text { session } / \mathrm{wk} \text { (group), } \\
10 \mathrm{wks}+40 \mathrm{~min} \times 6 \text { sessions } \\
\text { (individual)(Yoga) }\end{array}$ & $\begin{array}{l}\text { Simultaneous } \\
\text { (Usual treatment) }\end{array}$ & Mixed & (330 min) Yes & $70(9100 \mathrm{~min})$ & No treatment & Balance (BBS) \\
\hline $\begin{array}{l}\text { Yang \& Tang [31], } \\
\text { China }\end{array}$ & $\begin{array}{l}62(21 \%) \\
\text { MB: }: 32 \\
\text { CG: } 30 \\
\end{array}$ & $\begin{array}{l}\text { MB: } 51.43(15.63) \\
\text { CG: } 54.85(11.85)\end{array}$ & $\begin{array}{l}\text { MB: } 42.26(19.83) \mathrm{d} \\
\text { CG: } 40.57(23.12) \mathrm{d}\end{array}$ & $\begin{array}{l}\text { MB: } 20 / 8 \\
\text { CG: } 16 / 5\end{array}$ & $\begin{array}{c}40 \min \times 3 \text { sessions } / \mathrm{wk} \\
8 \text { wks (Tai Chi) }\end{array}$ & $\begin{array}{c}\text { Simultaneous } \\
\text { (general rehabilitation) }\end{array}$ & Group & (120 $\mathrm{min})$ No & $24(960 \mathrm{~min})$ & $\begin{array}{c}\text { General } \\
\text { rehabilitation }\end{array}$ & Balance (BBS) \\
\hline $\begin{array}{l}\text { Kim et al. [32], } \\
\text { Korea }\end{array}$ & $\begin{array}{l}24(8 \%) \\
\text { MB: } 12 \\
\text { CG: } 12 \\
\end{array}$ & $\begin{array}{l}\text { MB: } 53.45(11.54) \\
\text { CG: } 55.18(10.2)\end{array}$ & NR & NR & $\begin{array}{l}60 \mathrm{~min} \times 2 \text { sessions } / \mathrm{wk}, \\
6 \mathrm{wks} \text { (simplified Tai Chi) }\end{array}$ & $\begin{array}{c}\text { Simultaneous } \\
\text { (general rehabilitation } \\
\text { + physical therapy) }\end{array}$ & Group & $(120 \mathrm{~min})$ No & $12(720)$ & $\begin{array}{c}\text { General } \\
\text { rehabilitation }+ \\
\text { physical therapy }\end{array}$ & $\begin{array}{c}\text { Dynamic balance } \\
\text { (FRT) }\end{array}$ \\
\hline $\begin{array}{l}\text { Zhou, Li et al. [33], } \\
\text { China }\end{array}$ & $\begin{array}{l}22(0 \%) \\
\text { MB: } 11 \\
\text { CG: } 11\end{array}$ & $35-70$ & $<6 \mathrm{M}$ & NR & $\begin{array}{l}60 \mathrm{~min} \times 5 \text { sessions } / \mathrm{wk}, \\
4 \text { wks (Tai Chi exercise) }\end{array}$ & $\begin{array}{c}\text { Simultaneous } \\
\text { (device-assisted } \\
\text { rehabilitation) }\end{array}$ & NR & (300 $\mathrm{min})$ Yes & $20(1200 \mathrm{~min})$ & $\begin{array}{l}\text { Device-assisted } \\
\text { rehabilitation }\end{array}$ & Balance (BBS) \\
\hline $\begin{array}{l}\text { Yang et al. [38], } \\
\text { China }\end{array}$ & $\begin{array}{l}100(0 \%) \\
\text { MB: } 50 \\
\text { CG: } 50 \\
\end{array}$ & $\begin{array}{l}\text { MB: } 54.3(13.8) \\
\text { CG: } 55.2(14.6) \\
\end{array}$ & $\begin{array}{l}\text { MB: } 44.7(18.4) \mathrm{d} \\
\text { CG: } 42.6(16.7) \mathrm{d}\end{array}$ & $\begin{array}{l}\text { Unable to } \\
\text { identify }\end{array}$ & $\begin{array}{c}45 \text { min } \times 6 \text { sessions } / \text { wk, } \\
4 \text { wks (Tai Chi) }\end{array}$ & Separate & NR & (270 $\mathrm{min}$ ) Yes & $24(1080 \mathrm{~min})$ & $\begin{array}{l}\text { Exercise } \\
\text { rehabilitation }\end{array}$ & Balance (BBS) \\
\hline $\begin{array}{l}\text { Zhang et al. [39], } \\
\text { China }\end{array}$ & $\begin{array}{l}34(0 \%) \\
\text { MB: } 17 \\
\text { CG: } 17 \\
\end{array}$ & $\begin{array}{l}\text { MB: } 43.5(4.7) \\
\text { CG: } 42.8(5.1)\end{array}$ & $<6 \mathrm{M}$ & NR & $\begin{array}{c}60 \mathrm{~min} \times 5 \text { sessions } / \mathrm{wk} \\
4 \text { wks (Tai Chi) }\end{array}$ & Separate & Group & (300 $\mathrm{min})$ Yes & $20(1200 \mathrm{~min})$ & $\begin{array}{l}\text { General } \\
\text { rehabilitation }\end{array}$ & Balance (BBS) \\
\hline $\begin{array}{l}\text { Fu et al. }[40] \text {, } \\
\text { China }\end{array}$ & $\begin{array}{l}60(0 \%) \\
\text { MB: } 30 \\
\text { CG: } 30\end{array}$ & $\begin{array}{l}\text { MB: } 59.7(7.6) \\
\text { CG: } 60.3(8.4)\end{array}$ & $<3 \mathrm{M}$ & $\begin{array}{l}\text { MB: } 13 / 17 \\
\text { CG: } 10 / 20\end{array}$ & $\begin{array}{l}15 \mathrm{~min} \times 6 \text { sessions } / \text { wk, } \\
8 \text { wks (24-style Tai Chi) }\end{array}$ & $\begin{array}{l}\text { Simultaneous } \\
\text { (General } \\
\text { rehabilitation) }\end{array}$ & Individual & (90) No & $48(720 \mathrm{~min})$ & $\begin{array}{c}\text { General } \\
\text { rehabilitation }\end{array}$ & Balance (BBS) \\
\hline
\end{tabular}


Table 1. Cont.

\begin{tabular}{|c|c|c|c|c|c|c|c|c|c|c|c|}
\hline \multirow{2}{*}{$\begin{array}{l}\text { Reference } \\
\text { (Country) }\end{array}$} & \multicolumn{4}{|c|}{ Participant Characteristics } & \multicolumn{5}{|c|}{ Mind-Body Intervention } & \multirow{2}{*}{$\begin{array}{l}\text { Control Group } \\
\text { Activity }\end{array}$} & \multirow{2}{*}{$\begin{array}{l}\text { Outcomes } \\
\text { (Instrument }\end{array}$} \\
\hline & $\operatorname{ISZ}(\mathrm{AT})$ & $\begin{array}{c}\text { Mean Age or Age } \\
\text { Range (yr) }\end{array}$ & Course of Disease & $\begin{array}{l}\text { Ischemic/ } \\
\text { Hemorrhage }\end{array}$ & $\begin{array}{l}\text { Training Frequency and Length } \\
\text { (MB Component) }\end{array}$ & $\begin{array}{c}\text { Mode of } \\
\text { Combination }\end{array}$ & $\begin{array}{l}\text { Training } \\
\text { Mode }\end{array}$ & $\begin{array}{l}\text { (Training Dose) } \\
\geq 150 \text {-min weekly }\end{array}$ & $\begin{array}{l}\text { No. of Sessions } \\
\text { (Total Volume) }\end{array}$ & & \\
\hline $\begin{array}{l}\text { Liu et al. [41], } \\
\text { China }\end{array}$ & $\begin{array}{l}48(0 \%) \\
\text { MB: } 24 \\
\text { CG: } 24\end{array}$ & $\begin{array}{l}\text { MB: } 52.13(14.13) \\
\text { CG: } 53.51(12.63)\end{array}$ & $\begin{array}{l}\text { MB: } 17.65(5.34) \mathrm{d} \\
\text { CG: } 18.73(8.78) \mathrm{d}\end{array}$ & $\begin{array}{l}\text { MB: } 9 / 15 \\
\text { CG: } 8 / 16\end{array}$ & $\begin{array}{l}30 \mathrm{~min} \times \text { unclear, } 12 \mathrm{wks} \\
\text { (personalized Tai Chi) }\end{array}$ & $\begin{array}{l}\text { Simultaneous } \\
\text { (General } \\
\text { rehabilitation) }\end{array}$ & Individual & NR & NR & $\begin{array}{c}\text { General } \\
\text { rehabilitation }\end{array}$ & Balance (BBS) \\
\hline $\begin{array}{l}\text { Zhang, Li et al. [42], } \\
\text { China }\end{array}$ & $\begin{array}{l}62(0 \%) \\
\text { MB: } 31 \\
\text { CG: } 31 \\
\end{array}$ & $\begin{array}{l}\text { MB: } 55.07(4.81) \\
\text { CG: } 46.71(3.57)\end{array}$ & $\begin{array}{l}\text { MB: } 6.22(2.45) \mathrm{wk} \\
\text { CG: } 7.01(1.89) \mathrm{wk}\end{array}$ & $\begin{array}{l}\text { MB: } 21 / 10 \\
\text { CG: } 19 / 12\end{array}$ & $\begin{array}{l}40 \min \times 5 \text { sessions } / \text { wk, } \\
8 \mathrm{wks} \text { (Baduanjin Qigong) }\end{array}$ & $\begin{array}{c}\text { Simultaneous } \\
\text { (Usual treatment }+ \\
\text { balance training) }\end{array}$ & NR & (200 min) Yes & $40(1600 \mathrm{~min})$ & $\begin{array}{l}\text { Usual treatment }+ \\
\text { balance training }\end{array}$ & Balance (BBS) \\
\hline $\begin{array}{l}\text { Tian [43], } \\
\text { China }\end{array}$ & $\begin{array}{l}60(5 \%) \\
\text { MB: }: 30 \\
\text { CG: } 30 \\
\end{array}$ & $\begin{array}{l}\text { MB: } 54.3(4.7) \\
\text { CG: } 53(4.3)\end{array}$ & NR & $60 / 0$ & $\begin{array}{l}60 \mathrm{~min} \times 2 \text { sessions } / \mathrm{wk}, \\
12 \mathrm{wks} \text { (Baduanjin Qigong) }\end{array}$ & $\begin{array}{l}\text { Simultaneous } \\
\text { (Usual treatment) }\end{array}$ & Group & $(120 \mathrm{~min})$ No & $24(1440 \mathrm{~min})$ & Usual treatment & Balance (BBS) \\
\hline $\begin{array}{l}\text { Bai et al. [44], } \\
\text { China }\end{array}$ & $\begin{array}{l}60(0 \%) \\
\text { MB: }: 30 \\
\text { CG: } 30 \\
\end{array}$ & $\begin{array}{l}\text { MB: } 53.7(4.5) \\
\text { CG: } 51.3(7.5)\end{array}$ & $\begin{array}{l}\text { MB: } 43.2(6.53) \mathrm{d} \\
\text { CG: } 38.5(6.12) \mathrm{d}\end{array}$ & $\begin{array}{l}\text { MB: } 18 / 12 \\
\text { CG: } 19 / 11\end{array}$ & $\begin{array}{l}40 \mathrm{~min} \times 7 \text { sessions } / \mathrm{wk} \\
6 \mathrm{wks} \text { (Baduanjin Qigong) }\end{array}$ & $\begin{array}{l}\text { Simultaneous } \\
\text { (Balance training) }\end{array}$ & NR & (280 min) Yes & $42(1680 \mathrm{~min})$ & Balance training & Balance (BBS) \\
\hline $\begin{array}{l}\text { Zhou et al. [45], } \\
\text { China }\end{array}$ & $\begin{array}{l}68(0 \%) \\
\text { MB: }: 34 \\
\text { CG: } 34 \\
\end{array}$ & $\begin{array}{l}65.2(8.5) \text { for all } \\
\text { participants }\end{array}$ & NR & $0 / 68$ & $\begin{array}{l}\text { Unclear } \times 2 \text { sessions } / \text { wk, } \\
4 \text { wks (24-style Tai Chi) }\end{array}$ & $\begin{array}{c}\text { Simultaneous } \\
\text { (General } \\
\text { rehabilitation) }\end{array}$ & NR & NR & 8 (unclear) & $\begin{array}{l}\text { Drug treatment + } \\
\text { general } \\
\text { rehabilitation }\end{array}$ & Balance (BBS) \\
\hline $\begin{array}{l}\text { Zhang, Guo et al. [46], } \\
\text { China }\end{array}$ & $\begin{array}{c}224 \\
(1.3 \%) \\
\text { MB: } 115 \\
\text { CG: } 106\end{array}$ & 33 to 82 & 1 to 6 months & NR & $\begin{array}{l}40 \mathrm{~min} \times 7 \text { sessions } / \mathrm{wk} \\
6 \text { wks (Baduanjin Qigong) }\end{array}$ & $\begin{array}{l}\text { Simultaneous } \\
\text { (Bobath techniques }+ \\
\text { drug therapy) }\end{array}$ & NR & (280 $\mathrm{min}$ ) Yes & $24(960 \mathrm{~min})$ & $\begin{array}{l}\text { Bobath techniques } \\
+ \text { drug therapy }\end{array}$ & Balance (BBS) \\
\hline $\begin{array}{l}\text { Li et al. [47], } \\
\text { China }\end{array}$ & $\begin{array}{l}40(0 \%) \\
\text { MB: }: 20 \\
\text { CG: } 20\end{array}$ & $\begin{array}{l}\text { MB: } 57.8(7.3) \\
\text { CG: } 58.7(6.4)\end{array}$ & $\begin{array}{l}\text { MB: } 14.8(4.6) \mathrm{d} \\
\text { CG: } 14.5(4.5) \mathrm{d}\end{array}$ & $\begin{array}{l}\text { MB: } 13 / 7 \\
\text { CG: } 11 / 9\end{array}$ & $\begin{array}{c}40 \text { min } \times 7 \text { sessions } / \mathrm{wk}, \\
6 \text { wks (Tai Chi) }\end{array}$ & $\begin{array}{c}\text { Simultaneous } \\
\text { (General rehabilititation } \\
\text { + Prokin training) } \\
\end{array}$ & NR & $(280 \mathrm{~min})$ Yes & $42(1680 \mathrm{~min})$ & $\begin{array}{c}\text { General } \\
\text { rehabilitation }\end{array}$ & Balance (BBS) \\
\hline $\begin{array}{l}\text { Xu et al. [48], } \\
\text { China }\end{array}$ & $\begin{array}{l}80(0 \%) \\
\text { MB: } 40 \\
\text { CG: } 40\end{array}$ & $\begin{array}{l}\text { MB: } 60.14(10.25) \\
\text { CG: } 48.23(12.32)\end{array}$ & $\begin{array}{l}\text { MB: } 45.21(25.42) \mathrm{d} \\
\text { CG: } 47.34(22.56) \mathrm{d}\end{array}$ & $\begin{array}{l}\text { MB: } 18 / 22 \\
\text { CG: } 14 / 26\end{array}$ & $\begin{array}{l}40 \mathrm{~min} \times 7 \text { sessions } / \mathrm{wk}, \\
12 \text { wks (Tai Chi posture) }\end{array}$ & $\begin{array}{l}\text { Simultaneous } \\
\text { (General } \\
\text { rehabilitation) }\end{array}$ & Mixed & (280 $\mathrm{min}$ ) Yes & $84(3360 \mathrm{~min})$ & $\begin{array}{c}\text { General } \\
\text { rehabilitation }\end{array}$ & Balance (BBS) \\
\hline
\end{tabular}

Note: $\mathrm{ISZ}$ = initial sample size; $\mathrm{AT}=$ attrition rate; $\mathrm{wk}=$ week; $\mathrm{M}=$ month; $\mathrm{yr}=$ year; $\mathrm{MB}=$ mind-body exercise; $\mathrm{CG}=$ control group; $\mathrm{BBS}=$ The Berg Balance Scale; FRT = functional Reach

Test; TBT = Timed Balance Test; NR = Not Reported; mode of combination indicates whether mind-body movement intervention is combined with other treatments (drug therapy or usual

care). If only mind-body movement was used as the intervention program for stroke survivor, we considered "separate condition," vice versa. 


\subsection{Risk of Bias within Studies}

Table 2 summarizes the results of the methodological quality assessment. The summed scores of the PEDro scale across the eligible studies were relatively high, ranging from 5 to 9. Specifically, 13 studies fell between the range of 7-9 points, followed by 4 studies with 6 points, and one study with 5 points. Roughly $77.78 \%$ of the 18 RCTs selected did not clearly report use of concealed allocation and blinded assessors. The additional points that were deducted were due to high attribution rates [31], missing data management [29,30,32,43,46], between-group comparisons [28], and co-intervention equivalence [47].

Table 2. Summary of methodological quality for included studies.

\begin{tabular}{ccccccccccc}
\hline Author [Reference] & Item 1 & Item 2 & Item $\mathbf{3}$ & Item $\mathbf{4}$ & Item $\mathbf{5}$ & Item $\mathbf{6}$ & Item $\mathbf{7}$ & Item $\mathbf{8}$ & Item $\mathbf{9}$ & Score \\
\hline Taylor-Piliae et al. [27] & 1 & 1 & 1 & 1 & 1 & 1 & 1 & 1 & 1 & $9 / 9$ \\
Taylor-Piliae et al. [28] & 1 & 1 & 1 & 1 & 1 & 0 & 0 & 1 & 1 & $7 / 9$ \\
Schmid et al. [29] & 1 & 1 & 1 & 0 & 1 & 0 & 1 & 1 & 1 & $7 / 9$ \\
Immink et al. [30] & 1 & 1 & 1 & 1 & 1 & 0 & 1 & 1 & 1 & $8 / 9$ \\
Yang \& Tang [31] & 1 & 0 & 1 & 0 & 0 & 0 & 1 & 1 & 1 & $5 / 9$ \\
Kim et al. [32] & 1 & 0 & 1 & 0 & 1 & 0 & 1 & 1 & 1 & $6 / 9$ \\
Zhou et al. [33] & 1 & 0 & 1 & 0 & 1 & 1 & 1 & 1 & 1 & $7 / 9$ \\
Yang et al. [38] & 1 & 0 & 1 & 0 & 1 & 1 & 1 & 1 & 1 & $7 / 9$ \\
Zhang et al. [39] & 1 & 0 & 1 & 0 & 1 & 1 & 1 & 1 & 1 & $7 / 9$ \\
Fu et al. [40] & 1 & 0 & 1 & 1 & 1 & 1 & 1 & 1 & 1 & $8 / 9$ \\
Liu et al. [41] & 1 & 0 & 1 & 0 & 1 & 1 & 1 & 1 & 1 & $7 / 9$ \\
Zhang et al. [42] & 1 & 0 & 1 & 0 & 1 & 1 & 1 & 1 & 1 & $7 / 9$ \\
Tian [43] & 1 & 0 & 1 & 0 & 1 & 0 & 1 & 1 & 1 & $6 / 9$ \\
Bai et al. [44] & 1 & 0 & 1 & 0 & 1 & 1 & 1 & 1 & 1 & $7 / 9$ \\
Zhou et al. [45] & 1 & 0 & 1 & 0 & 1 & 1 & 1 & 1 & 1 & $7 / 9$ \\
Zhang et al. [46] & 1 & 0 & 1 & 0 & 1 & 0 & 1 & 1 & 1 & $6 / 9$ \\
Li et al. [47] & 1 & 0 & 1 & 0 & 1 & 1 & 1 & 1 & 0 & $6 / 9$ \\
Xu et al. [48] & 1 & 0 & 1 & 0 & 1 & 1 & 1 & 1 & 1 & $7 / 9$ \\
\hline
\end{tabular}

Note: Item 1 = randomization; Item 2 = concealed allocation; Item $3=$ similar baseline; Item $4=$ blinding of assessors; Item $5=$ more than $85 \%$ retention; Item $6=$ missing data management (intention-to-treat analysis); Item 7 = between-group comparison; Item $8=$ point measure and measures of variability; Item $9=$ Co-intervention (should be either avoided in the trial design or similar between the index and control groups); $1=$ explicitly described and present in details; $0=$ absent, inadequately described, or unclear; NA = not applicable.

\subsection{Effects of MBM on Improvement of Balance}

The Egger's regression test was not significant (Egger's regression intercept $=4.679, p=0.172$ ), along with a relatively symmetrical Funnel plot (Figure 2). The included studies used three different instruments (the Berg Balance Scale, the functional Reach Test, and the Timed Balance Test) to examine the effects of the MB exercise intervention on balance function in comparison to a control group. A higher positive value for all the balance tests indicated better balance performance. The aggregated results demonstrated a significant benefit of MBM interventions on balance function: (Hedge's $g=1.59$, CI 0.98 to $2.19, p<0.001, I^{2}=94.95 \%$; Figure 3).

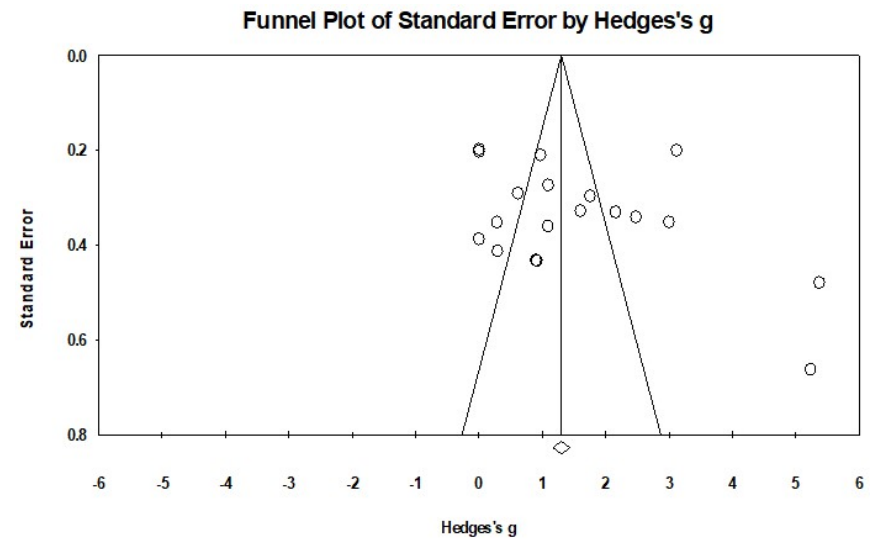

Figure 2. The Funnel Plot of included dependent effect sizes $(k=18)$. 


\section{Balance: Mind-body exercises vs Control}

\begin{tabular}{|c|c|c|c|c|c|}
\hline \multirow[t]{3}{*}{ Study name } & \multicolumn{5}{|c|}{ Statistics for each study } \\
\hline & \multicolumn{5}{|c|}{ Hedges'sL ower Upper } \\
\hline & $g$ & limit & & Z-Value & p-Value \\
\hline Bai et al (2011) & 2.48 & 1.81 & 3.15 & 7.28 & 0.00 \\
\hline Tian (2017) & 2.16 & 1.51 & 2.81 & 6.53 & 0.00 \\
\hline Zhang et al (2013) & 3.12 & 2.73 & 3.51 & 15.60 & 0.00 \\
\hline Zhang et al (2016) & 1.76 & 1.18 & 2.34 & 5.93 & 0.00 \\
\hline T aylor-P iliae (2014) 1 & 0.00 & -0.39 & 0.39 & 0.00 & 1.00 \\
\hline T aylor-P iliae (2014) 2 & 0.00 & -0.40 & 0.40 & 0.00 & 1.00 \\
\hline Fu et al (2016) & 1.09 & 0.56 & 1.63 & 3.99 & 0.00 \\
\hline Xu et al (2014) & 5.37 & 4.43 & 6.31 & 11.22 & 0.00 \\
\hline Zhou et al (2010) & 3.00 & 2.31 & 3.69 & 8.53 & 0.00 \\
\hline Yang et al (2013) & 0.97 & 0.56 & 1.39 & 4.64 & 0.00 \\
\hline Yang \& T ang (2016) & 0.61 & 0.04 & 1.18 & 2.11 & 0.03 \\
\hline Immink et al (2014) & 0.29 & -0.51 & 1.10 & 0.71 & 0.48 \\
\hline Schmid et al (2012) & 0.29 & -0.40 & 0.97 & 0.81 & 0.42 \\
\hline Kim et al (2015) & 0.90 & 0.05 & 1.74 & 2.08 & 0.04 \\
\hline Liu et al (2009) & 1.61 & 0.96 & 2.25 & 4.90 & 0.00 \\
\hline T aylor-Piliae et al (2011), & 0.00 & -0.76 & 0.76 & 0.00 & 1.00 \\
\hline Zhou et al (2015) & 0.92 & 0.07 & 1.77 & 2.12 & 0.03 \\
\hline Li et al (2014) & 5.23 & 3.94 & 6.53 & 7.90 & 0.00 \\
\hline Zhang et al (2017) & 1.09 & 0.38 & 1.79 & 3.03 & 0.00 \\
\hline & 1.59 & 0.98 & 2.19 & 5.11 & 0.00 \\
\hline
\end{tabular}

Hedges's g and $95 \% \mathrm{CI}$
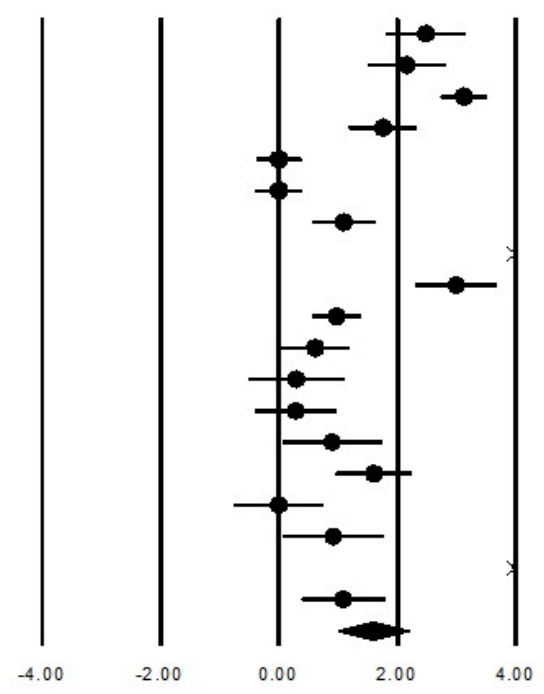

\section{Control Mind-body}

Figure 3. Effect of mindfulness-based movement intervention on balance function.

\subsection{Moderator Analysis}

The results of the sub-group analyses are displayed in Table 3. There were significant effects for training frequency $(\mathrm{Q}[1]=4.69, p=0.03)$, session length $(\mathrm{Q}[1]=14.21, p=0.00)$, and mode of combination $(\mathrm{Q}[1]=7.59, p=0.01)$ on balance function. On the other hand, no significant effects were found for intervention duration $(\mathrm{Q}[1]=0.07, p=0.79)$, weekly training duration $(\mathrm{Q}[1]=0.88, p=0.35)$, or sample size $(Q[1]=0.91, p=0.34)$. In addition, the results of the meta-regression indicated that the total number of sessions ( $\beta=0.00142,95 \%$ CI 0.0039 to $0.0244, p=0.0067$ ) and dose of weekly training ( $\beta=0.00776,95 \%$ CI 0.00579 to $0.00972, p=0.00$ ) had significantly positive effects on balance function. 
Table 3. Moderator analysis for mind-body exercises versus control group.

\begin{tabular}{|c|c|c|c|c|c|c|c|c|}
\hline \multirow{2}{*}{ Categorical Moderator } & \multirow{2}{*}{ Level } & \multirow{2}{*}{ No. of Studies } & \multirow{2}{*}{ Hedge's $g$} & \multirow{2}{*}{$95 \% \mathrm{CI}$} & \multirow{2}{*}{$I^{2}, \%$} & \multicolumn{3}{|c|}{ Test for between-Group Homogeneity } \\
\hline & & & & & & Q-Value & $\mathrm{df}(\mathrm{Q})$ & $p$-Value \\
\hline \multirow{2}{*}{ Intervention duration } & $<12$ weeks & 13 & 1.64 & 0.92 to 2.36 & $92.74 \%$ & 0.07 & 1 & 0.79 \\
\hline & $\geq 12$ weeks & 6 & 1.47 & 0.41 to 2.52 & $96.67 \%$ & & & \\
\hline \multirow{2}{*}{ Training frequency } & $<5$ sessions/week & 9 & 0.94 & 0.14 to 1.74 & $91.94 \%$ & 4.69 & 1 & 0.03 \\
\hline & $\geq 5$ sessions/week & 10 & 2.17 & 1.4 to 2.93 & $94.76 \%$ & & & \\
\hline \multirow{2}{*}{ Session length } & Less than $45 \mathrm{~min}$ & 8 & 2.54 & 1.81 to 3.27 & $94.94 \%$ & 14.21 & 1 & 0.00 \\
\hline & 45 min or longer & 10 & 0.66 & 0.01 to 1.3 & $81.79 \%$ & & & \\
\hline \multirow{2}{*}{ Mode of combination } & Separate & 5 & 0.41 & -1.15 to -0.80 & $79.98 \%$ & 7.59 & 1 & 0.01 \\
\hline & Simultaneous & 14 & 2.01 & 1.41 to 2.6 & $93.63 \%$ & & & \\
\hline \multirow{2}{*}{ Weekly training dose $\geq 150$-min } & Yes & 12 & 1.71 & 0.91 to 2.51 & $96.45 \%$ & 0.88 & 1 & 0.35 \\
\hline & No & 5 & 1.01 & -0.22 to 2.24 & $78.18 \%$ & & & \\
\hline \multirow{2}{*}{ Sample size } & $<60$ & 8 & 1.23 & 0.26 to 2.19 & $87.96 \%$ & 0.91 & 1 & 0.34 \\
\hline & $\geq 60$ & 11 & 1.84 & 1.03 to 2.64 & $96.56 \%$ & & & \\
\hline Continuous Moderator & No. of Studies & $\beta$ & \multicolumn{2}{|c|}{ 95\% Confidence Interval } & \multicolumn{2}{|c|}{ Q-Value } & $\mathrm{df}$ & $p$ \\
\hline Number of total sessions & 17 & 0.0142 & \multicolumn{2}{|c|}{0.0039 to 0.0244} & \multicolumn{2}{|c|}{7.36} & 1 & 0.0067 \\
\hline Weekly training dose & 17 & 0.00776 & \multicolumn{2}{|c|}{0.00579 to 0.00972} & \multicolumn{2}{|c|}{59.62} & 1 & 0.000 \\
\hline
\end{tabular}

Note: mode of combination indicates whether mind-body movement intervention is combined with other treatments (drug therapy or usual care). If only mind-body movement was used as the intervention program for stroke survivor, we considered "separate condition," vice versa. 


\section{Discussion}

Our review critically evaluated and statistically synthesized available evidence on the effects of MBM (Tai Chi, Qigong and yoga) on balance function among stroke survivors. Based on the evidence available from previous literature, our review suggests that implementing these exercises may improve balance function among stroke survivors. Moreover, data from the emerging literature has increasingly highlighted the efficacy of augmentative Tai Chi or Qigong training for patients recovering from the acute phase of a stroke. Given previous concerns regarding the efficacy and safety of yoga as a stroke rehabilitation treatment due to a lack of information, these findings are encouraging [49]. To our knowledge, this is the first meta-analysis that has examined the effects of MBM interventions on post-stroke rehabilitation. The findings from this study are also significant from a public health perspective given that many stroke survivors experience varying degrees of balance loss, which then affects their mobility, physical functioning, quality of life, mood and independence [50]. Given these findings, MBM may be implemented as a complementary treatment to stroke patients as a safe and inexpensive option to aid in achieving more favorable outcomes.

Presently, the exact mechanisms through which Tai Chi, Qigong and yoga affect balance in stroke survivors are unknown. However, one modern theory suggests that these MBM elicit positive changes by enhancing physiological proprioception - specifically by pairing posture, movement and breath control practices with a special state of awareness. This enhanced state of awareness thereby improves and strengthens the overall state of vegetative regulation, also known as homeostasis, which may mediate the positive outcomes associated with these MBM [51].

Overall, there are many advantages of employing MBM as an add-on therapy for post-stroke patients. It is easy to learn, has few known side effects and is widely accessible to people of all ages and physical strength. In addition, these MBM facilitate relaxation and personal integration aspects (e.g., an enhanced ability to sustain attention [52]), which contribute to increased mindful awareness and personal acceptance [53]. These integration methods may be beneficial for patients and may ultimately transcend post-stroke treatment and elicit positive outcomes in other areas of the patients' lives, as well. However, some disadvantages are that qualified Tai Chi, Qigong and yoga instructors may not be available in some areas thus limiting accessibility. Furthermore, long-term adherence of these practices tends to be low which may reduce the overall efficacy and feasibility of these practices.

One major strength of this review is that it included published empirical studies in both English and Chinese. This expansive inclusion is appropriate and important since most of the current studies assessing the rehabilitative effects of MBM for balance of stroke patients were conducted in China and were published mostly in the Chinese language. Therefore, by including articles in Chinese, the findings from researchers published in Chinese peer-reviewed journals are included and acknowledged. Other strengths of this meta-analysis include: the use of a standardized scale to assess the methodological quality of the studies, the use of a recognized meta-analytic method to evaluate the magnitude of the MB exercises' intervention effect (pooled effect size), the variations in the frequency and duration of meditative movements practice (moderator analyses), and the extent of symmetry of the effect sizes (funnel plot and Egger's regression) on balance function.

This review is not without limitations even if encouraging findings are found. First, Tai Chi, Qigong and yoga in this review are formulated into MBM and their quantitative data on balance are synthesized. While key researchers in MB research propose that Tai Chi, Qigong and yoga can all be viewed as meditative movements given that they use similar techniques [54], further studies are needed to discern whether there are mechanistic differences between these practices and confirm that these exercises have comparable effects for post-stroke rehabilitation. Secondly, many of the studies used non-blinded assessors, so a subjectivity and social desirability bias may influence the interpretation of these research findings. Third, most of the studies did not offer MBM as a monotherapy, but as an alternative treatment to the interventions already received by the patients. Therefore, it may be difficult to conclude whether the positive outcomes were attributed to stand-alone MBM, a synergetic intervention effect, or the treatment already being received by the patients. Nonetheless, our data 
provides support for MBM as an alternative treatment for stroke survivors to improve their balance function. Another limitation was that, across all studies, numerous different interventions were received by the control groups which made the interpretations and comparisons of outcomes difficult. Additionally, the frequency and the duration of the MBM practices varied extensively between the different studies which further complicated our analyses. For example, in the moderator analyses, while higher training frequency, mode of combination, session length, weekly training dosage, and total number of sessions were associated with better outcomes, a longer intervention program ( $\geq 12$ weeks) and longer weekly training intervention ( $\geq 150 \mathrm{~min}$ ) were not. Such findings are hard to make specific recommendations regarding the dosage of the intervention. Therefore, future studies should be conducted to further establish effective parameters for treatment and define the critical point at which the duration of treatment becomes ineffective. Other limitations included the fact that most of the studies were conducted in Asia, therefore it remains unclear whether the findings are generalizable to non-Asian populations. Interestingly, the four studies that were performed in Western countries all showed negative outcomes, while the remaining studies conducted in non-Western countries showed positive outcomes [27-30]. Therefore, these findings raise the question of generalizability and whether the interventions could be comparable between different regions. Lastly, given that studies reporting positive or significant results are more likely to be published and outcomes are that are statistically significant have greater likelihood of being reported, this may lead to publication bias.

\section{Conclusions}

The findings of this study suggest that Tai Chi, Qigong or yoga could be used as an alternative treatment for post-stroke patients to improve balance function. There were significant weaknesses in the design of the analyzed studies and the outcomes varied in different regions. Future studies with more robust methodology will be needed to provide a more definitive conclusion.

Author Contributions: L.Z. and L.W. contributed to the conception and design of the review. L.Z. applied the search strategy. L.Z., N.Z. and L.W. applied the selection criteria. L.Z., N.Z. and L.W. completed assessment of risk of bias. All authors analyzed the data and interpreted data. L.Z. and A.Y. wrote this manuscript. All authors edited this manuscript. L.Z. is responsible for the overall project.

Funding: Research on Wushu Overseas Communication Application Framework Based on Audience Exchange (17YJA890025); the Fundamental Research Funds for the Central Universities (WUT: 2018VI014).

Acknowledgments: This study is supported by Humanities and Social Sciences of Ministry of Education Planning. Conflicts of Interest: No conflict of interest existed in this study.

\section{References}

1. Hall, M.; DeFrances, C. Hospitalization for stroke in U.S. hospitals, 1989-2009. Diabetes 2012, $18,23$.

2. Mackay, J.; Mensah, G.A.; Greenlund, K. The Atlas of Heart Disease and Stroke; World Health Organization: Geneva, Switzerland, 2004.

3. Lu, J.; Xu, L.; Zhai, Y. Direct economic burden of cerebrovascular disease, during 1993-2008 in China. Chin. J. Epidemiol. 2014, 35, 1263-1266.

4. Brønnum-Hansen, H.; Davidsen, M.; Thorvaldsen, P. Long-term survival and causes of death after stroke. Stroke 2001, 32, 2131-2136. [CrossRef] [PubMed]

5. Geurts, A.; de Haart, M.; van Nes, I.; Duyseans, J. A review of standing balance recovery from stroke. Gait Posture 2005, 22, 267-281. [CrossRef] [PubMed]

6. Weerdesteyn, V.; de Niet, M.; van Duijnhoven, H. Falls in individuals with stroke. J. Rehabil. Res. Dev. 2008, 45, 1195-1213. [CrossRef] [PubMed]

7. Kerse, N.; Parag, V.; Feigin, L.; McNaughton, H.; Hackett, L.; Bennett, A.; Anderson, S.; Auckland, R. Falls after stroke: Results from the Auckland Regional Community Stroke (ARCOS) Study, 2002 to 2003. Stroke 2008, 39, 1890-1893. [CrossRef] [PubMed]

8. Kochanek, K.; Murphy, S.; Arias, E. Mortality in the United States, 2013; National Center for Health Statistics: Hyattsville, MD, USA, 2014. 
9. Mozaffarian, D.; Benjamin, E.J.; Go, A.S.; Arnett, D.K.; Blaha, M.J.; Cushman, M.; Das, S.R.; de Ferranti, S.; Després, J.-P.; Fullerton, H.J.; et al. Heart Disease and Stroke Statistics-2016 Update: A Report From the American Heart Association. Circulation 2016, 133, e38-e360. [CrossRef] [PubMed]

10. Hatem, M.; Saussez, G.; Della Faille, M.; Prist, V.; Zhang, X.; Dispa, D.; Bleyenheuft, Y. Rehabilitation of motor function after stroke: A multiple systematic review focused on techniques to stimulate upper extremity recovery. Front. Hum. Neurosci. 2016, 10, 442. [CrossRef] [PubMed]

11. Van Peppen, R.; Kwakkel, G.; Wood-Dauphinee, S.; Hendriks, J.; Van der Wees, J.; Dekker, J. The impact of physical therapy on functional outcomes after stroke: What's the evidence? Clin. Rehabil. 2004, 18, 833-862. [CrossRef] [PubMed]

12. Godwin, M.; Wasserman, J.; Ostwald, K. Cost associated with stroke: Outpatient rehabilitative services and medication. Top. Stroke Rehabil. 2011, 1, 676-684. [CrossRef] [PubMed]

13. Zou, L.; Sasaki, E.; Zeng, N.; Wang, C.; Sun, L. A systematic review with meta-analysis of mindful exercises on rehabilitative outcomes among poststroke patients. Arch. Phys. Med. Rehabil. 2018, 18, 30283-30291. [CrossRef] [PubMed]

14. Zou, L.; Wang, C.; Tian, Z.; Wang, H.; Shu, Y. Effect of Yang-Style Tai Chi on Gait Parameters and Musculoskeletal Flexibility in Healthy Chinese Older Women. Sports 2017, 5, 52. [CrossRef] [PubMed]

15. Zou, L.; Wang, C.; Yeung, A.; Talwar, S.; Wang, C.; Liu, Y.; Shu, Y.; Thomas, A. A Review Study on the beneficial effects of Baduanjin. J. Altern. Complement. Med. 2017, 24, 324-335. [CrossRef] [PubMed]

16. Yu, H.; Wang, D. Tai Chi exercise and hemiplegia rehabilitation. Chin. J. Rehabil. Theory Pract. 2002, 18, 447-448.

17. Zou, L.; SasaKi, J.; Wang, H.; Xiao, Z.; Fang, Q.; Zhang, M. A Systematic Review and Meta-Analysis Baduanjin Qigong for Health Benefits: Randomized Controlled Trials. Evid. Based Complement. Altern. Med. 2017. [CrossRef] [PubMed]

18. Zou, L.; Wang, H.; Xiao, Z.; Fang, Q.; Zhang, M.; Li, T.; Du, G.; Liu, Y. Tai chi for health benefits in patients with multiple sclerosis: A systematic review. PLoS ONE 2017, 12, e0170212. [CrossRef] [PubMed]

19. Zou, L.; Wang, H.; Li, T.; Lu, M.; Wang, C. Effect of Health-Qigong on spinal mobility and disease activity in people with ankylosing spondylitis. Trav. Hum. 2017, 80, 1585-1597.

20. Zou, L.; Wang, C.; Chen, K.; Shu, Y.; Chen, X.; Luo, L.; Zhao, X. The Effect of Taichi practice on attenuating bone mineral density loss: A systematic review and meta-analysis of randomized controlled trials. Int. J. Environ. Res. Public Health 2017, 14, 1000. [CrossRef] [PubMed]

21. Taylor-Piliae, E.; Hoke, M.; Hepworth, T.; Latt, D.; Najafi, B.; Coull, M. Effect of Tai Chi on physical function, fall rates and quality of life among older stroke survivors. Arch. Phys. Med. Rehabil. 2014, 95, 816-824. [CrossRef] [PubMed]

22. Taylor-Piliae, E.; Coull, M. Community-based Yang-style Tai Chi is safe and feasible in chronic stroke: A pilot study. Clin. Rehabil. 2012, 26, 121-131. [CrossRef] [PubMed]

23. Schmid, A.; Van Puymbroeck, M.; Altenburger, A.; Schalk, L.; Dierks, A.; Miller, K.; Damush, M.; Ravata, M.; Williams, S. Post-stroke balance improves with yoga: A pilot study. Stroke 2012, 43, 2402-2407. [CrossRef] [PubMed]

24. Immink, A.; Hillier, S.; Petkov, J. Randomized controlled trial of yoga for chronic poststroke hemiparesis: Motor function, mental health, and quality of life outcomes. Top. Stroke Rehabil. 2014, 21, 256-271. [CrossRef] [PubMed]

25. Yang, H.; Tang, Q. Effects of Tai Chi Quan on motor function among stroke survivors. Chin. J. Rehabil. Med. Res. 2016, 31, 1146-1148.

26. Kim, H.; Kim, Y.; Lee, S. Effects of therapeutic Tai Chi on balance, gait, and quality of life in chronic stroke patients. Int. J. Rehabil. Res. 2015, 38, 156-161. [CrossRef] [PubMed]

27. Zhou, L.; Li, Z.; Zhou, Y. Preliminary study on the effect of modified Tai Chi for motor function in patients with stroke. Chin. J. Integr. Med. 2015, 13, 878-880.

28. Ding, M. Tai Chi for stroke rehabilitation: A focused review. Am. J. Phys. Med. Rehabil. 2012, 91, 1091-1096. [CrossRef] [PubMed]

29. Taylor-Piliae, E.; Haskell, L. Tai Chi exercise and stroke rehabilitation. Top. Stroke Rehabil. 2007, 14, 19-22. [CrossRef] [PubMed] 
30. Lazaridou, A.; Phibrook, P.; Tzika, A. Yoga and mindfulness as therapeutic interventions for stroke rehabilitation: A systematic review. Evid. Based Complement. Altern. Med. 2013, 2013, 357108. [CrossRef] [PubMed]

31. Lynton, H.; Kligler, B.; Shiflett, S. Yoga in stroke rehabilitation: A systematic review and results of a pilot study. Top. Stroke Rehabil. 2007, 14, 1-8. [CrossRef] [PubMed]

32. Moher, D.; Liberati, A.; Tetzlaff, J.; Altman, G. Preferred reporting items for systematic reviews and meta-analyses: The PRISMA statement. Ann. Intern. Med. 2009, 151, 264-269. [CrossRef] [PubMed]

33. Kachan, D.; Olano, H.; Tannenbaum, L.; Annane, W.; Mehta, A.; Arheart, L.; Fleming, E.; Yang, X.; McClure, A.; Lee, J. Prevalence of mindfulness practices in the US Workforce: National health interview survey. Prev. Chronic Dis. 2017, 14, e01. [CrossRef] [PubMed]

34. US Department of Health and Human Services. Physical Activity Guidelines Advisory Committee Report. 2008. Available online: https://health.gov/paguidelines/report/pdf/committeereport.pdf (accessed on 26 May 2018).

35. Zou, L.; Yeung, A.; Quan, X.; Hui, S.; Hu, X.; Chan, M.; Wang, C.; Boyden, D.; Sun, L.; Wang, H. Mindfulness-based Baduanjin exercise for depression and anxiety in people with physical or mental illnesses: A Systematic Review and Meta-analysis of randomized controlled trials. Int. J. Environ. Res. Public Health 2018, 15, 321. [CrossRef] [PubMed]

36. Zou, L.; Yeung, A.; Quan, X.; Boyden, D.; Wang, H. A Systematic review and Meta-analysis of Mindfulness-based (Baduanjin) exercise for alleviating musculoskeletal pain and improving sleep quality in people with chronic diseases. Int. J. Environ. Res. Public Health 2017, 15, 206. [CrossRef] [PubMed]

37. Harvey, R.L.; Macko, R.F.; Stein, J.; Winstein, C.J.; Zorowitz, R.D. Stroke Recovery and Rehabilitation, 2nd ed.; Demos Medical Publishing: New York, NY, USA, 2008.

38. Yang, Z.; Liu, D.; Chang, Y. The therapeutic effects of TaiChi balance training for stroke with balance disorders. Contemp. Med. 2013, 19, 5-6.

39. Zhang, G. Analysis on the effect of modified Taijiquan on stroke patients in rehabilitation of movement function. J. Nurs. 2017, 6, 17-21.

40. Fu, C.; Zhang, Q. Effects of Taijiquan on balance function and walking ability of stroke hemiplegic patients in convalescent phase. J. Rehabil. Med. 2016, 31, 536-539.

41. Liu, T.; Qin, P.; Chen, X. Effect of Tai Chi on balance function in post-stroke patients. Chin. J. Phys. Med. Rehab. 2009, 31, 781-782.

42. Zhang, Y.; Li, L. Treatment of 31 cases of balance function disorder after cerebral stroke by eight-section brocade and balance training. Shandong J. Chin. Med. 2016, 35, 716-718.

43. Tian, H. Effects of Baduanjn Qigong on motor function among stroke survivors: A randomized controlled trial. World Latest Med. Inf. 2017, 17, 251-252.

44. Bai, Y.; Mao, H.; Guo, J. Research on Baduanjin combined with function training in improving balance functions of stroke patients. China J. Chin. Med. 2011, 26, 1231-1232.

45. Zhou, Q.; Xu, J.; Hu, A. Observation on recover of cerebral infraction patients by TaijiQuan training. Chin. J. Pract. Nerv. Dis. 2010, 13, 20-22.

46. Zhang, M.; Guo, J.; Bai, Y. Baduanjin combined with rehabilitation program for balance function in patients with stroke. LishiZhen Med. Mater. Med. Res. 2013, 24, 2438-2439.

47. Li, H.; Zhang, G. Effect of Tai Chi Quan footwork combined with Prokin balance apparatus on balance dysfunction of patients with hemiplegia due to cerebral stroke. ShanXi J. Tradit. Chin. Med. 2014, 30, 10-12.

48. Xu, X.; Bai, J.; Zhang, H. Analysis of curative effect for Tai Chi on the balance function of patients with hemiplegia after stroke. Heibei J. Tradit. Chin. Med. 2014, 36, 1149-1151.

49. Lawrence, M.; Celestino Junior, T.; Matozinho, H.; Govan, L.; Booth, J.; Beecher, J. Yoga for stroke rehabilitation. Cochrane Database Syst. Rev. 2017. [CrossRef] [PubMed]

50. Saunders, D.H.; Sanderson, M.; Hayes, S.; Kilrane, M.; Greig, C.A.; Brazzelli, M.; Mead, G.E. Physical fitness training for stroke patients. Cochrane Database Syst. Rev. 2016. [CrossRef] [PubMed]

51. Matos, C.; Sousa, M.; Gonçalves, M.; Gabriel, J.; Mahado, J.; Greten, J. Qigong as a traditional vegetative biofeedback therapy: Long-term conditioning of physiological mind-body effects. Biomed. Res. Int. 2015, 2015, 531789. [CrossRef] [PubMed] 
52. Oken, S.; Zajdel, D.; Kishiyama, S.; Flegal, K.; Dehen, C.; Haas, M.; Kraemer, F.; Lawrence, J.; Leyva, J. Randomized, controlled, six-month trial of yoga in healthy seniors: Effects on cognition and quality of life. Altern. Ther. Health Med. 2006, 12, 40-47. [PubMed]

53. Garrett, R.; Immink, A.; Hillier, S. Becoming connected: The lived experience of yoga participation after stroke. Disabil. Rehabil. 2011, 33, 2404-2415. [CrossRef] [PubMed]

54. Payne, P.; Crane-Godreau, A. Meditative movement for depression and anxiety. Front. Psychiatry $2013,4,71$. [CrossRef] [PubMed]

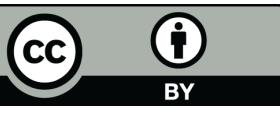

(c) 2018 by the authors. Licensee MDPI, Basel, Switzerland. This article is an open access article distributed under the terms and conditions of the Creative Commons Attribution (CC BY) license (http://creativecommons.org/licenses/by/4.0/). 